
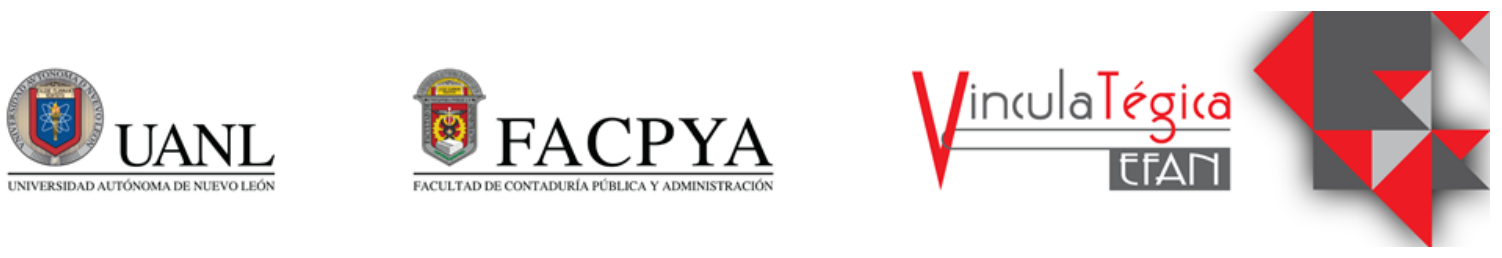

\title{
RSE y ODS como herramientas del crecimiento en PyMEs: un estudio comparativo
}

\author{
Ma. Teresa de la Luz Sainz Barjas ${ }^{1}$, Juan José Chiñas Valencia² y María Guadalupe \\ Aguirre Alemán ${ }^{3}$
}

${ }^{1}$ Universidad Veracruzana campus Coatzacoalcos (México), tsainz@uv.mx, Dirección: Av. Universidad km. 7.5 Coatzacoalcos, Veracruz, México. CP 96538, Teléfono:921 2115700 Ext. 55711

${ }^{2}$ Universidad Veracruzana campus Coatzacoalcos (México), jchinas@uv.mx, Dirección: Av. Universidad km. 7.5 Coatzacoalcos, Veracruz, México. CP 96538, Teléfono:921 2115700 Ext. 55711

${ }^{3}$ Universidad Veracruzana campus Coatzacoalcos (México), gaguirre@uv.mx, Dirección: Av. Universidad km. 7.5 Coatzacoalcos, Veracruz, México. CP 96538, Teléfono:921 2115700 Ext. 55711

Información del artículo revisado por pares

Fecha de aceptación: junio-2021

Fecha de publicación en línea: diciembre-2021

DOI: https://doi.org/10.29105/vtga7.1-107

\section{Resumen}

El crecimiento empresarial es detonante para el desarrollo de los países, las empresas en la actualidad se han alineado a los ODS y la RSE como herramientas que potencializan su capacidad hacia generar dicho crecimiento. En el presente estudio se tiene como objetivo general comparar el nivel en que las PyMEs comerciales y de servicios del sur del estado Veracruz que realizan acciones alineadas a los ODS y RSE, está contribuyendo al crecimiento empresarial. La investigación se posiciona desde el paradigma empírico-analítico, con un enfoque cuantitativo y transversal. Para la recolección de la información se diseñó una encuesta basada en la revisión de la literatura y la cual fue validada, además se recurrió a un muestreo no probabilístico a conveniencia, obteniendo dos muestras independientes: 75 comerciales y 34 de servicios, los informantes fueron gerentes o dueños. El método de análisis utilizado fue el análisis descriptivo y se recurrió a la prueba de hipótesis a través de la técnica de U de Mann-Whitney. Los resultados demuestran que existen diferencias entre ambas muestras, obteniendo un nivel tanto en comerciales y de servicios medio-alto, no obstante, se observaron diferencias, la prueba de hipótesis denota que se apoya la hipótesis de investigación, en donde se apoya que el nivel en el crecimiento entre ambas muestras es diferente. En definitiva, las acciones que adopta las PyMEs sobre ODS y RSE si están impactando en el

\section{Abstract}

Business growth is a trigger for the development of countries, companies today have aligned themselves with the ODS and RSE as tools that enhance their capacity to generate said growth. The general objective of this study is to compare the level at which commercial and service SMEs in the southern state of Veracruz that carry out actions aligned with the ODS and RSE, are contributing to business growth. The research is positioned from the empirical-analytical paradigm, with a quantitative and transversal approach. To collect the information, a survey was designed based on the literature review and which was validated, in addition, a non-probabilistic convenience sampling was used, obtaining two independent samples: 75 commercial and 34 from services, the informants were managers or owners. The analysis method used was descriptive analysis and hypothesis testing was used through the Mann-Whitney U technique. The results show that there are differences between both samples, obtaining a level both in commercial and medium-high services, however, differences were observed, the hypothesis test denotes that the research hypothesis is supported, where it is supported that the level the growth between the two samples is different. In short, the actions that SMEs take on ODS and RSE are having an impact on business growth in the market where they are developed. 
crecimiento empresarial en el mercado donde se desarrollan.

Palabras clave: RSE, ODS, PyMEs

\section{INTRODUCCIÓN}

La Responsabilidad Social Empresarial (RSE), está viviendo una transición relevante en el contexto corporativo, debido a que ahora es considerada como una herramienta de gestión empresarial, que plantea la inclusión de la RSE en la declaración de la misión, visión y objetivos, con el propósito de que las entidades no se conformen con tan solo ofrecer productos y servicios de calidad, sino con pretender ser una gran empresa que hace un mundo mejor.

En este mismo tenor, derivado de una propuesta de la ONU, surgen los objetivos de desarrollo sostenible (ODS), los cuales conforman el primer marco de consenso integral histórico que busca terminar con los principales problemas de índole social, medioambiental y económica de la sociedad.

Este binomio, está invitando a las organizaciones a adoptar prácticas que coadyuben en su crecimiento empresarial, desde una óptica socialmente responsable.

Sin embargo, esta demanda, ha sido percibida por empresas grandes y en menor o nula escala, por las pequeñas y medianas empresas (PyMEs).

Es por ello, que la presente tiene como objetivo primordial comparar el nivel en que las PyMEs comerciales y de servicios del sur del estado Veracruz que realizan acciones alineadas a los ODS y RSE, están contribuyendo al crecimiento empresarial.

\subsection{Antecedentes}

El tema de RSE ha tomado un importante valor en las últimas décadas, por tanto, existen gran variedad de investigadores que realizan estudios para abonar en la literatura y generación de conocimiento contemporáneo en esta propuesta. A continuación, se mencionan algunos antecedentes importantes que fundamentan este estudio:
Keywords: CSR, SDG, sme

JEL: M14, O44, R11

\section{Internacionales}

- En 2013, en Colombia se llevó a cabo un diagnóstico de la situación real con respecto a las prácticas de RSE en 30 PyMEs de las cinco principales ciudades: Barranquilla, Bogotá, Cali, Cartagena y Medellín, con el objetivo de generar una propuesta de manual para que los empresarios puedan dar sus primeros pasos en la incorporación de la RSE de una forma metodológicamente organizada y sencilla, buscando, no el cumplimiento de requisitos legales y normativos, sino la competitividad y sostenibilidad de las empresas. El instrumento estuvo conformado por las dimensiones dirección y gobierno corporativo; derechos humanos y organización interna; bienes y servicios; proveedores y relaciones comerciales; medio ambiente y apoyo a la comunidad. Los hallazgos mostraron que la RSE en las PyMEs se encuentra en una etapa incipiente, donde es evidente el desconocimiento en el tema, y las políticas están enfocadas en los intereses económicos como la rentabilidad y la competitividad que les garanticen continuar en la lucha por sobrevivir (Flores, 2013).

- Herrera, Larrán, Lechuga y Martínez (2016), llevaron a cabo una investigación exploratoria con PyMEs de España, con la finalidad de analizar el nivel de desarrollo y de desempeño que pueden tener en relación con las actividades de RSE, así como indagar sobre su correlación con la diversidad de género, nivel de formación del propietario/gerente, estructura de la propiedad, el tamaño y el sector. Las resultas, permitieron aseverar que las empresas cuentan con una actitud positiva 
hacia la RSE, debido a que desempeñan acciones socialmente responsables reflejadas en la formación del gerente/propietario, el tamaño y el sector.

\section{Nacionales}

- Labelle y Navarrete (2018), se dieron a la tarea de llevar a cabo una investigación en 200 PyMEs de Jalisco, México y 200 de Quebec, Canadá, las cuales se caracterizaron por coincidir en las reglas institucionales surgidas del TLCAN. El objetivo consistió en cotejar la presencia de un efecto de convergencia e isomorfismo en prácticas de RSE. Se aplicó un instrumento desarrollado para tal efecto y los resultados fueron sometidos a pruebas ANOVA para confirmar si hay aproximaciones isomorfas o no de cada variable analizada. Las resultas permitieron exponer que existen similitudes sobre las prácticas de RSE en las PyMEs de ambos países; que las PyMEs mexicanas son más proactivas en materia de RSE que las canadienses y se llegó a la conclusión de que las hipótesis planteadas no permitieron comprender el fenómeno de la influencia de los tratados entre ambos países sobre las prácticas de RSE.

- En la zona costa de Baja California, Avendaño, Sierra, y Lobo (2017) efectuaron una investigación para realizar una estimación de la adopción de acciones de RSE en empresas hortícolas de exportación. Esta, se realizó en dos etapas, la primera, consistió en el cálculo del índice de adopción de innovaciones en RSE a través de cuatro categorías de acciones: capital humano, sustentabilidad de la producción agrícola, uso del agua y fuentes de energía y ética empresarial. En la segunda fase, se efectuó un ajuste del Indicador Ethos para PyMEs, sugerido por el Instituto Argentino de Responsabilidad Social Empresarial, el cual tiene como meta, identificar la situación de las empresas en cuanto a la implementación de RSE y las áreas de oportunidad para la mejora en la gestión empresarial. Finalmente, se dedujo que las áreas de ética de la empresa y uso eficiente del agua y la energía son las que presentan el mayor reto para la implementación de protocolos de RSE.

\section{MARCO TEÓRICO}

El prohijamiento de prácticas de RSE no es de carácter obligatorio y puede parecer que está más ligada a las grandes empresas; sin embargo, en las PyMEs es más genuino su empleo debido a la estrecha relación con su contexto y contigüidad con sus clientes. Por otra parte, es resulta inconcebible que puedan existir empresas exitosas en sociedades fracasadas, es por ello preponderante el amalgamado de la RSE y los ODS en las labores habituales de las PyMEs.

\subsection{Conceptualización de la RSE}

La RSE es el compromiso voluntario de tomar decisiones, medidas, actividades y acciones éticas y transparentes con el fin de determinar los problemas de interés público (medio ambiente, pobreza, desigualdad de ingresos, atención de salud, hambre, desnutrición y analfabetismo), con el fin de ayudar a la organización (empresa, estado, universidad, etc.), tanto en lo interno como en lo externo, considerando las dimensiones fundamentales que comprende el concepto de sustentabilidad (económica, ambiental y social) de todos sus grupos de interés (stakeholders: empleados, socios, clientes, comunidades locales, medioambiente, accionistas, proveedores, etc.), demostrando respeto por la sociedad, los valores y el medio ambiente, favoreciendo así a alcanzar una excelencia empresarial matizada por un desarrollo social y ambiental sostenible y económicamente viable (De Miguel, 2011; Avendaño y William, 2013; Cajiga, 2009; y Viteri, 2010).

\subsection{Beneficios de la RSE en PyMEs}

Los beneficios que la RSE puede generar en las PyMEs que adopten sus prácticas, se clasifican en tres ámbitos, el primero, hace 
referencia a la cuestión laboral, reflejándose en la mejora en el clima laboral, debido al incremento en la motivación y productividad, retención del talento humano, en la disminución de los errores, ausentismo y rotación. La segunda vertiente, está representada por los beneficios económicos, tales como la reducción de costos operativos, ahorro por el consumo eficiente de recursos, prosperidad en el desempeño financiero y competitividad, por ende, atracción de financiamiento. La tercera esfera, personifica a los privilegios estratégicos, tales como la mejora en la gestión, el ímpetu a la gestión del riesgo, lealtad de consumidores, excelente imagen de marca, adherencia de nuevos clientes, proactividad frente a las demandas de los socios comerciales, relaciones a largo plazo con proveedores, y restricción de los riesgos de comunicación (Enguix, 2021).

\subsection{Principios básicos para desarrollar una estrategia de RSE en la pyme}

Una maniobra holística orientada a la RSE debe estar sustentada en tres componentes (De Miguel, 2011):

- Pensamiento estratégico. Cada pyme debe realizar un ejercicio de autodiagnóstico, con el objeto de redefinir su misión y visión, detección de recursos disponibles y de los grupos de interés que favorecerán en la obtención de una ventaja competitiva y entonces estará en posibilidad de seleccionar las tareas a ejecutar en materia de RSE.

- Acción. Cada una de las decisiones a ejercer, deberán ser planeadas, tangibles, dirigidas a un grupo de interés definido e incorporar a todas las personas de la organización.

- Comunicación. Es indispensable compartir de manera eficaz los resultados de las acciones llevadas a cabo en materia de RSE con los grupos de interés, con la sociedad y con otras PyMEs y conocer la percepción de estos.

Cabe mencionar que este tripié, debe tener como ejes rectores el desarrollo económico, el bienestar social y el respeto al medio ambiente, es decir, debe comulgar en su totalidad con la sustentabilidad. Adicional y en este mismo camino, el gobierno de la empresa debe adoptar una actitud basada en los principios de visión a largo plazo; diversidad en cuanto a opiniones culturas, perspectivas, edades, sexo, desde la perspectiva interna y externa de la organización; apertura al diálogo con las partes interesadas; integridad y responsabilidad (Ricart y Rodríguez, 2005).

\subsection{Análisis de Debilidades, Amenazas, Fortalezas y Oportunidades (DAFO) de la RSE en PyMEs}

Estudios llevados a cabo por Urriolagoitia, Murillo y Lozano (2009), en países europeos como Italia, Gran Bretaña y España, indican que las PyMEs desde hace algunos años, de manera inherente perpetran quehaceres alineados con la RSE tales como la formación continua, seguridad laboral, participación en proyectos en pro de la comunidad, promoción de iniciativas culturales, control de impacto medio ambiental, inclusión de personas discapacitadas y comercio justo; sin embargo, todo este esfuerzo no ha tenido el impacto suficiente en el mundo empresarial, debido en gran medida por la inadecuada comunicación de dichas acciones, porque padecen de desinformación, de conciencia y recursos; porque sufren para emprender y adoptar acciones de RSE con un enfoque más estratégico que incremente su competitividad. En ese sentido, plantean Roser, Cabrerizo, De la Cuesta y Vázquez (2005) el análisis DAFO mostrado en la Tabla 1 que ilustra los aspectos de responsabilidad social corporativa (RSC) clasificados de tal manera que puedan apoyar a las PyMEs en el diseño de un plan de acción al respecto.

\begin{tabular}{cc}
\multicolumn{2}{c}{ Tabla 1. Análisis DAFO de RSC en PyMEs } \\
\hline Debilidades & Amenazas \\
\hline Recursos limitados & Sanciones \\
\hline
\end{tabular}




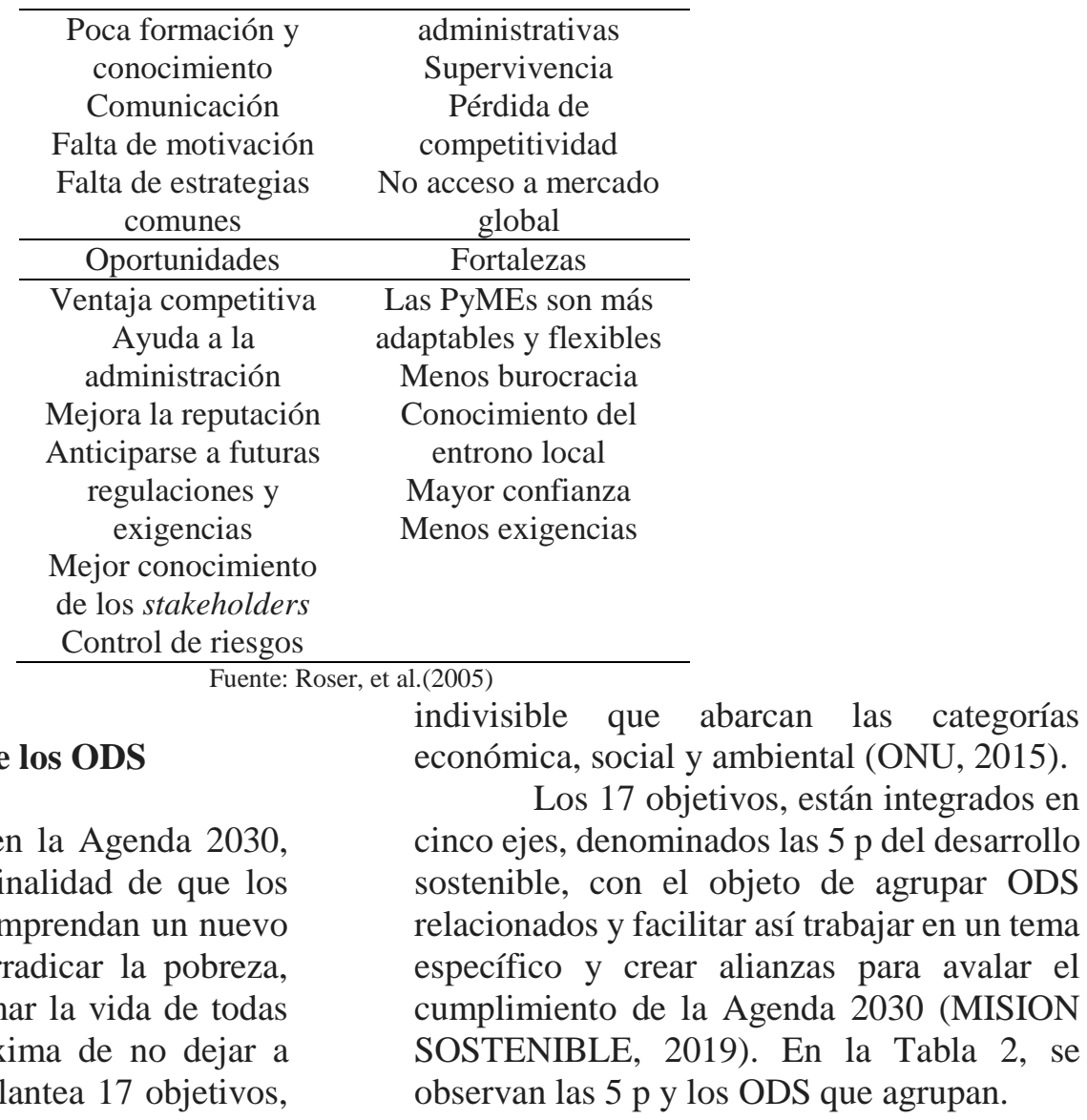

\subsection{Conceptualización de los ODS}

Los O están integrados en la Agenda 2030, fueron gestados con la finalidad de que los países y sus sociedades emprendan un nuevo trayecto que atestigüe erradicar la pobreza, proteger el planeta, optimar la vida de todas las personas, con la máxima de no dejar a nadie atrás. La Agenda plantea 17 objetivos, con 169 metas de carácter integrado e

Tabla 2. Las 5 p del desarrollo sostenible con sus respectivos ODS

\begin{tabular}{cc}
\hline $5 \mathrm{p}$ & Objetivos de \\
desarrollo sostenible \\
ODS 1: fin de la \\
pobreza \\
ODS 2: hambre cero \\
ODS 3: salud y \\
bienestar \\
people (personas) \\
OD 4: educación de \\
calidad \\
ODS 5: igualdad de \\
género \\
\hline ODS 7: energía \\
asequible y no \\
contaminante \\
ODS 8: trabajo \\
decente y crecimiento \\
económico \\
ODS 9: industria, \\
innovación e \\
infraestructura \\
ODS 10: reducción \\
de las desigualdades \\
ODS 11: ciudades y \\
comunidades \\
sostenibles \\
\hline
\end{tabular}




\begin{tabular}{cc}
\hline & ODS 6: agua limpia y \\
& saneamiento \\
& ODS 12: producción \\
& y consumo \\
& responsable \\
planet (planeta) & ODS 13: acción por \\
el clima & ODS 14: vida \\
& submarina \\
& ODS 15: vida de \\
& ecosistemas terrestres \\
\hline peace (paz) & ODS 16: paz, justicia \\
e instituciones sólidas \\
\hline ODS 17: alianzas \\
partnership (alianzas) & para lograr los \\
& objetivos
\end{tabular}

Fuente: elaboración propia con información de MISIONSOSTENIBLE (2019)

\subsection{Importancia de los ODS para las empresas}

Como ya se mencionó, los ODS fueron gestados para suscitar la colaboración entre actores y movilizar los bríos a nivel global alrededor del cúmulo de metas comunes, es por ello que las empresas como miembros de la sociedad, tienen el deber ético de contribuir al florecimiento social (personas, planeta y prosperidad), que redundará en beneficios tales como mercados estables, regulados y competitivos; sistemas financieros transparentes e instituciones libres de corrupción y bien gobernadas; materias primas y energía accesibles, consumidores con poder adquisitivo; empleados calificados; alianzas público-privadas para el desarrollo; y el fomento de cadenas de valor integradas (Remacha, 2017).

\subsection{La guía para la acción empresarial en los ODS}

La guía para la acción empresarial en los ODS, también conocida como SDG (Sustainable Development Goal) Compass, o la brújula de los ODS, mediante cinco pasos, ayuda a que las empresas entiendan, gestionen, alineen sus estrategias y midan su contribución con los ODS. Está vinculada con la RSE, debido a que invita a las empresas a cumplir con la legislación pertinente, a respetar los estándares internacionales mínimos y a abordar de manera prioritaria todos los impactos negativos sobre los derechos humanos. Los cinco pasos son: (GRI [Global Reporting Initiative]/United Nations Global Compact/wbesd [World Business Council for Sustainable Development], 2016; Remacha, 2017)

- Entendiendo los ODS. Consiste en familiarizarse con ellos y entender las oportunidades y responsabilidades que cada uno de ellos simbolizan para el negocio.

- Definiendo prioridades. No todos los ODS son igual de importantes para la empresa, por ello es imperante definir las prioridades de la empresa y así poder enfocar los esfuerzos y beneficiarse de las oportunidades y desafíos.

- Estableciendo objetivos. Los objetivos de sostenibilidad deben ser específicos, medibles y con límites de tiempo, con el propósito de incentivar prioridades compartidas e incitar el rendimiento de toda la organización.

- Integrando. Es crucial conformar a la sostenibilidad en el quehacer esencial de la empresa e incorporar metas a través de las funciones para abordar los ODS y trasmutar así la oferta de productos y servicios, segmentos de clientes, gestión de la cadena de suministro, la elección y el uso de materias primas, las redes de transporte y distribución y fin de vida útil del producto.

- Reportando y comunicando. Es relevante que la empresa divulgue a los grupos de 
interés la incorporación de los ODS en su quehacer cotidiano y el impacto de dicho evento.

\subsection{Escenario de las PyMEs en México}

CEPAL (2021), testifica que las PyMEs a nivel nacional o regional, en países industrializados o en aquellos con inferior nivel de crecimiento, cuentan con una importante notabilidad en la economía y la esfera del personal ocupado. El acceso a mercados, tecnologías y capital humano; así como la casi nula vinculación con sus símiles, que incide en su productividad, capacidad de exportación y potencial de crecimiento, retratan sus peculiaridades.

Alrededor del 69\% de las PyMEs, están dirigidas por hombres y, por lo tanto, el $31 \%$ restante, por mujeres; el $45 \%$ de ellas, han conseguido perseverar activas por lo menos 12 años y el 75\% están constituidas bajo la estructura de propiedad familiar (FEADPYME, 2018).

Con base a lo establecido en el Estudio sobre la Demografía de los Negocios (EDN) 2020, del mes de mayo de 2019 a septiembre de 2020, 213,998 PyMEs bajaron sus cortinas de manera definitiva, en contraste, tan solo hubo 17,406 nacimientos; hecho que sin lugar a dudas, refleja de manera fehaciente el contexto que las PyMEs están enfrentando, distinguido por retos que las invitan a reflexionar sobre cómo reingeniar su negocio y detectar áreas de oportunidad para alcanzar la competitividad, rentabilidad y eficiencia en el corto, mediano y largo plazo (INEGI, 2021).

Por otro lado, la Encuesta sobre el Impacto Generado por COVID-19 en las Empresas (ECOVID-IE) 2020, realizada a empresas grandes, PyMEs y micros, de los sectores industrial, comercio y servicios, indicó que las principales afectaciones pandémicas fueron: $80.7 \%$ de las PyMEs sufrieron una disminución en los ingresos, el $65.2 \%$ experimentó baja demanda y el $27.9 \%$ de ellas enfrentó escasez de insumos. Asimismo, el $77.5 \%$ implementó campañas de información y comunicación virtuales como medida para enfrentar las afectaciones de la pandemia. Además, los resultados muestran que $34.2 \%$ de las PyMEs se retrasaron en el pago de sus deudas. En cuanto a la operación del negocio, el $46.1 \%$ de las PyMEs adoptaron las ventas por Internet, $24.9 \%$ ofertaron nuevos bienes y servicios (INEGI, 2020).

Sin duda alguna, el entorno actual que enfrenta las PyMEs abre las posibilidades a la reinvención, a transitar hacia nuevas formas de negocio como las ventas por Internet, hacia la diversificación ofertando nuevos productos y servicios, y adoptar formas de trabajo flexible que fomente el empleo por turnos y el home office. Además, este renacer las ha hecho revalorar el uso de tecnologías innovadoras como una fuerza resiliente para mantenerse vigentes ante la fuerte competitividad del mercado.

Ante este escenario desalentador Esemanal (2021) y la GPTWR (2020), proponen condiciones fundamentales que favorecerán a las PyMEs en la solvatación de su situación actual:

- Capacitación en función de las áreas de oportunidad y de los requerimientos de formación,

- Cultura laboral más humana,

- Reinvención de estrategias comerciales,

- Mejora en tres ejes: gente, procesos y tecnología,

- Conocimiento de los compradores, y

- Disciplina y planeación financiera

Es así como, la época actual ha marcado a la actividad económica y empresarial en todo el mundo, por lo que las empresas (incluyendo a las PyMEs), deben tomar decisiones difíciles. En este sentido la visión debe dirigirse a cuatro aspectos fundamentales: (Canal, 2020)

1. El negocio. Prevalecerá la eficiencia operativa, el manejo responsable de los recursos, la resiliencia y calidad como ejes rectores de la sustentabilidad de la empresa, desarrollados en un marco de buena gobernanza, ética y transparencia.

2. La gente. El cuidado de los colaboradores será fundamental durante y después de la pandemia. Las empresas deberán procurar el empleo, el ingreso, la salud y el bienestar de su elemento humano. 
3. Los clientes. Es momento de fortalecer los vínculos con los clientes, procurando su bienestar y fortaleciendo el sentido de pertenencia. Las PyMEs deberán ser capaces apoyar y aportar valor a su relación con los clientes.

4. La sociedad. Cada empresa deberá analizar qué puede aportar a la sociedad en tiempos difíciles y contribuir al bien común. Crear sinergias e impactar en el empoderamiento y desarrollo de la comunidad.

Como puede observarse, en las propuestas de solución antes mencionadas, las prácticas de RSE, alineadas con los ODS se encuentran de manera inherente inmersas en ellas.

\section{Método}

La presente investigación se plantea desde el enfoque empírico analítico y su alcance es transversal-correlacional (Ortín, 2015), con un tratamiento de datos para estudios cuantitativos no experimental (Hernández, Fernández y Baptista, 2014). La recolección de la información se realizó a través de la técnica de encuesta y el procesamiento de los datos se llevó acabo en el software estadístico SPSS versión 25 .

\subsection{Planteamiento del problema}

El crecimiento empresarial es un factor detonante para el desarrollo de los países, por tanto, es importante que las naciones establezcan políticas públicas enfocadas a mejorar las condiciones de estos contextos empresariales. Por un lado, la Agenda 2030 que establece los ODS ha puesto de manifiesto el rol protagónico que juegan las empresas hacia el desarrollo sostenible, por otro lado, la RSE que se ha caracterizado por actividades que realizan las empresas para coadyuvar a resolver problemas sociales, económicos y medio ambientales, es de suma importancia para el equilibro entre el consumo de las empresas y su contribución a los problemas del contexto.

La PyMEs en México son fundamentales para el crecimiento económico. De acuerdo con el informe del Senado de la República para 2020, las PyMEs generan 72\% de los empleos en México, además de que, cumplen un rol detonante para la inclusión social y son un eslabón en la cadena de actividades económicas $\mathrm{y}$, sobretodo indispensables para el desarrollo regional y local (Senado de la República, 2020).

Las políticas públicas que se han diseñado en los últimos años han adoptado la visión de los ODS, asumiendo la responsabilidad de todos deben contribuir a lograr la sostenibilidad. En el caso de las PyMEs, estas han adoptado las políticas orientadas a contribuir al desarrollo sostenible, que a través de diversas acciones las ponen práctica en sus actividades cotidianas y que además con el pensamiento de empresa socialmente responsable han institucionalizado en sus políticas internas esta filosofía.

Entendiendo que las PyMEs realizan diversas acciones y prácticas asociadas a los ODS y RSE, y que algunas hasta desconocen que están realizando actividades alineadas a las políticas, es necesario conocer la complejidad de los diversos escenarios a los que estas se enfrentan ante los cambios disruptivos y si el aplicar la antes acciones están contribuyendo al crecimiento empresarial.

El sur del Estado de Veracruz es bien conocido por su clúster industrial que en gran medida contribuye al desarrollo económico, sin embargo, en la región existen otras diversas PyMEs comerciales y de servicios que son detonantes de la economía local y regional, y de donde existe un gran número de empleos.

La PyMEs del sur del estado de Veracruz en sintonía con las políticas públicas de adoptar estrategias para contribuir a los ODS y con las prácticas de RSE en su cotidianidad contribuyen al crecimiento empresarial. De lo antes expuesto, considerando comparar las PyMEs comerciales y de servicios surge el planteamiento: $¿$ Existen diferencias entre el nivel de contribución al crecimiento empresarial entre las PyMEs comerciales y de servicios?

\subsection{Objetivos}


Para el desarrollo de esta investigación se planteado como objetivo general:

- Comparar el nivel de contribución al crecimiento empresarial entre las PyMEs comerciales y de servicios del sur del estado de Veracruz.

\subsection{Hipótesis}

Ho: "El nivel de contribución al crecimiento empresarial no es diferente entre PyMEs comerciales y de servicios"

H1: "El nivel de contribución al crecimiento empresarial es diferente entre PyMEs comerciales y de servicios"

\subsection{Análisis de la muestra}

Para el calculo de la muestra se recurrió a un muestreo de tipo no probabilístico que combinó el intencional y a conveniencia (Otzen y Manterola, 2017), obteniendo la participación de 109 sujetos de estudio distribuidos en dos muestras: 34 de empresas de servicios y 75 comerciales. Como se mencionó con antelación se aplicó una encuesta diseñada y validada a través de la revisión por expertos y la estadística, en la Tabla 3, se menciona la distribución.

El cuestionario, está compuesto de 63 ítems en una escala de tipo ordinal, las preguntas fueron diseñadas desde la teoría de los ODS y la RSE, se enlazan de forma que los resultados mostrarán el nivel que contribución al crecimiento empresarial. Por tanto, en la prueba piloto que se realizó previamente se obtuvo un 0,992 que de acuerdo Celia y Arias (2005) es excelente.

Tabla 3. Alfa de Cronbach

\begin{tabular}{|c|c|c|}
\hline & Dimensión & ítem \\
\hline D1 & Gestión global & $1-21$ \\
\hline D2 & Competitividad & $22-28$ \\
\hline D3 & Relaciones & $29-38$ \\
\hline D4 & Impactos & $39-50$ \\
\hline \multirow[t]{2}{*}{ D5 } & Transparencia & $51-63$ \\
\hline & Total & 63 \\
\hline
\end{tabular}

La aplicación de la encuesta se llevó a cabo a través de Google drive, durante el periodo de agosto-octubre de 2020. Al obtener la respuesta de los gerentes o dueños de las PyMEs se procedió a procesar los datos en una base de datos que posteriormente se proceso en el software SPSS versión 25.

En la Tabla 4, se aprecian los resultados de identificación de los encuestados, con relación al sexo, en el caso de las comerciales son mujeres $(53,3 \%)$ las que en su mayoría están al frente de estas, sin embargo, en el caso de servicios existe una distribución equitativa. Respecto, al grado de escolaridad, en las comerciales en su mayoría $(49,3 \%)$ solo tienen bachillerato y las de servicios licenciatura $(55,9 \%)$.

\begin{tabular}{|c|c|c|c|}
\hline & Componente & Comerciales & Servicios \\
\hline \multirow{3}{*}{ Sexo } & Hombre & 46,7 & 50 \\
\hline & Mujer & 53,3 & 50 \\
\hline & Total & 100,0 & 100,0 \\
\hline \multirow{4}{*}{ Grado } & Bachillerato & 49,3 & 26.5 \\
\hline & Licenciatura & 34,7 & 55.9 \\
\hline & Posgrado & 16,0 & 17.6 \\
\hline & Total & 100,0 & 100,0 \\
\hline \multirow{3}{*}{ Puesto } & Dueño & 49,3 & 44,1 \\
\hline & Gerente & 50,7 & 55,9 \\
\hline & Total & 100,0 & 100,0 \\
\hline \multirow{5}{*}{ Edad } & $17-30$ años & 44,0 & 35,1 \\
\hline & 31-40 años & 15,9 & 29,2 \\
\hline & 41-50 años & 18,6 & 26,3 \\
\hline & 50 años o más & 21,3 & 8,8 \\
\hline & Total & 100,0 & 100,0 \\
\hline
\end{tabular}


Fuente: elaboración propia.

Por otro lado, respecto al papel del rol de los encuestados en las PyMEs, en ambos sectores son gerentes los que están a cargo, respecto a la edad, sucede lo mismo, la mayoría se encuentra en un intervalo de edad entre 17-30 años, lo cual denota que estás PyMEs están siendo administradas por personal joven.

En la Tabla 5, se aprecian los resultados sobre datos de identificación de las empresas. Con relación al origen de su propiedad, en el caso de las comerciales en su mayoria $(57,5 \%)$ provienen del familiar y en las de servicios la mayoria $(58,8 \%)$ son no familiar, respecto al número de empleados, en ambos sectores oscila entre 11-50 colaboradores.

Tabla 5. Datos de empresa

\begin{tabular}{cccc}
\hline & Componente & Comerciales & Servicios \\
\hline \multirow{3}{*}{ Propiedad } & Familiar & 57,5 & 41,2 \\
& No familiar & 42,5 & 58,8 \\
& Total & 100,0 & 100,0 \\
& $11-50$ & 92,8 & 82,0 \\
Empleados & $51-200$ & 7,2 & 18,0 \\
& Total & 100,0 & 100,0 \\
& $1-10$ años & 61,5 & 73,5 \\
Antigüedad & $11-20$ años & 24,1 & 8,8 \\
& 21 años o & 14,6 & 17,4 \\
& más & 100,0 & 100,0 \\
\hline & Total & 100
\end{tabular}

Por lo que respecta a la antigüedad de las PyMEs, en ambos sectores tienen de 1-10 años en el mercado. En definitiva, en ambos sectores se encuentran diferencias, por tanto, se analizará si dichas diferencias son significativas en el grado que contribuyen al crecimiento empresarial.

\subsection{Resultados}

A continuación, se presentan los resultados de

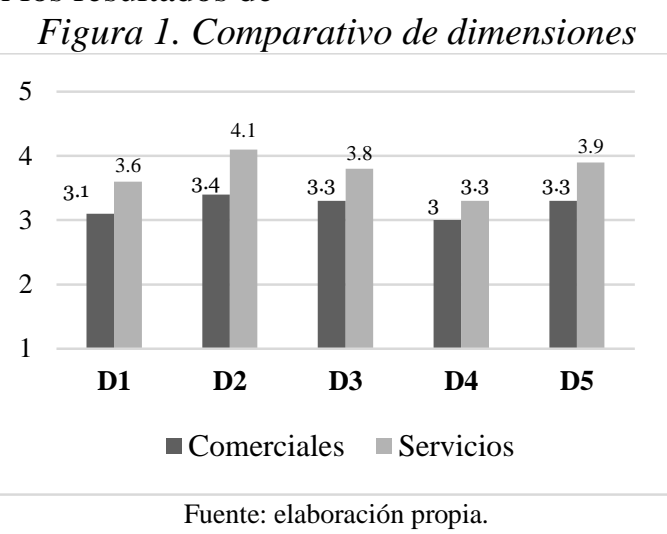

considerando la escala utilizada, se puede

Por otro lado, en la D2 que evalúa la competitividad, en la que posee un mayor nivel, lo cual denota que la PyMEs se encuentran en constante competencia entre ellas. Sin embargo, comparando los niveles y la investigación, en la Figura 1, se aprecian los promedios de cada dimensión de estudio. Se hace el comparativo entre ambos sectores, en genera se observa que las PyMEs de servicios son las que han estado realizando prácticas sobre ODS y RSE, por lo cual, han contribuido en un nivel mayor al crecimiento empresarial. Por otro lado, en ambos sectores respecto al impacto han realizado en menor medida, aún así las de servicio poseen un mayor nivel respecto de las comerciales. afirmar qué, aunque existen diferencias entre las empresas, en cada una de las dimensiones de estudio se aprecia que todas se encuentran por encima de la media, lo cual indica que estas PyMEs si contribuyen al crecimiento 
empresarial.

contribuyen al crecimiento empresarial.

Corroborando lo anterior, en la Tabla

6 , se presenta el nivel en que estás empresas

\begin{tabular}{ccccc}
\multicolumn{3}{c}{ Tabla 6. Nivel de incidencia-comparativo } \\
\hline \multirow{2}{*}{ Variable } & \multicolumn{2}{c}{ Comerciales } & \multicolumn{3}{c}{ Servicios } \\
& $\%$ & Nivel* & $\%$ & Nivel* $^{*}$ \\
\hline D1 & 62,0 & 2 & 72,0 & 2 \\
D2 & 68,0 & 2 & 82,0 & 1 \\
D3 & 66,0 & 2 & 76,0 & 2 \\
D4 & 60,0 & 2 & 66,0 & 2 \\
D5 & 66,0 & 2 & 78,0 & 2 \\
General & 64,4 & 2 & 74,8 & 2 \\
*Niveles de aplicación: 1 (alto), 2 (medio-alto), 3 \\
(medio), 4 (medio-bajo), 5 (bajo) \\
\hline \multicolumn{5}{c}{ Fuente: elaboración propia. }
\end{tabular}

Para la asignación de valores se recurrió al método de análisis de porcentaje, dando un valor para considerar el nivel en que contribuye al crecimiento empresarial. Tanto en las PyMEs comerciales y de servicio el grado en el que contribuye se encuentra en un nivel medio-alto, lo cual denota que estas empresas aplican los ODS y la RSE y su contribución al crecimiento es significativa. En la Figura 2, se aprecia el nivel de aplicación de la RSE y ODS respecto a cada ítem del estudio.

Figura 2. Nivel de cada ítem

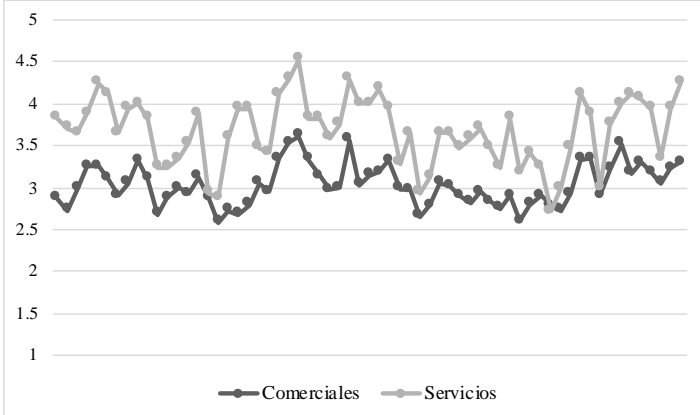

Fuente: elaboración propia.

El análisis por cada dimensión e ítem permite visualizar que existen diferencias entre los niveles obtenidos en ambas muestras, pero considerando la hipótesis de investigación, es necesario determinar si esas diferencias

son

estadísticamente
Tabla 7. Prueba de normalidad

\begin{tabular}{|c|c|c|c|}
\hline \multirow{2}{*}{ Sector } & \multicolumn{3}{|c|}{ Kolmogorov-Smirnova } \\
\hline & Estadístico & $\mathrm{gl}$ & Sig. \\
\hline \multirow[t]{3}{*}{ Comercial } & 0.169 & 75 & 000 \\
\hline & \multicolumn{3}{|c|}{ Shapiro-Wilk } \\
\hline & $\begin{array}{l}\text { Estadístico } \\
0.787\end{array}$ & $34^{\mathrm{gl}}$ & $\begin{array}{l}\text { Sig. } \\
000\end{array}$ \\
\hline
\end{tabular}

prueba de Kolmogorov-Smirnova y Shapiro-

Considerando que la muestra de las comerciales es 75 informantes y en el caso de las comerciales 34 , se recurrió a calcular la Wilk (Berlanga y Rubio, 2006), obteniendo de ello qué, en el caso de las comerciales un Pvalor de 000 y en el caso de servicios 000 , se significativas. Para ello, se recurrió a la prueba de normalidad (Tabla 7) para conocer el comportamiento de los datos y decidir la técnica por la cual se contrastará la hipótesis de investigación. 
denota que la distribución de los datos es no normal y, por lo tanto, la hipótesis se analizará con pruebas de tipo no paramétrica.

Teniendo en consideración lo anterior, y analizando que el objetivo es comparar las dos muestras, el tipo de escala es ordinal, y que los datos presentan un comportamiento no normal, se recurre a la prueba de U de MannWhitney, con la finalidad de conocer si existen diferencias estadísticamente significativas entre ambas muestras, en la Tabla 8 , se observa la prueba de hipótesis.

Tabla 8. Prueba de hipótesis

\begin{tabular}{lc}
\hline & Incidencia de los ODS y RSE \\
\hline U de Mann-Whitney & .000 \\
W de Wilcoxon & 820 \\
$\mathrm{Z}$ & -7.483 \\
Sig. asintótica(bilateral) & .000 \\
\hline Fuente: elaboración propia. &
\end{tabular}

La prueba indica que la U de MannWhitney obtenida es de .000 y un P-valor de 0.000 , lo cual denota que se rechaza la hipótesis nula, entendiendo de esta manera que si existen diferencias significativas entre ambas muestras. Estos resultados señalan que el análisis estadístico se corrobora con la prueba de hipótesis, y se pone de manifiesto la hipótesis de investigación acerca de que existe diferencias en el nivel de contribución al crecimiento empresarial entre las PyMEs comerciales y de servicios.

\section{CONCLUSIONES}

En esta investigación se diseñó y aplicó una encuesta a gerentes y/o dueños, de PyMEs ubicadas al sur del estado de Veracruz, de la cual se obtuvieron dos muestras independientes: 75 comerciales y 34 de servicios. En la cual se buscó comparar el nivel de contribución al crecimiento empresarial entre las PyMEs comerciales y de servicios. El método de análisis básicamente consistió comparar el nivel de ambas muestras con estadística descriptiva y posterior, se realizó la prueba de hipótesis para contrastar la establecida con los resultados.

El análisis descriptivo permitió identificar que las empresas de servicios contribuyen en mayor medida al crecimiento empresarial y las de servicios, aunque en menor medida, su contribución es significativa. Además, de acuerdo con el método utilizado se determinó que ambas muestras se encuentran en un nivel medio-alto en su contribución al crecimiento.

Por lo que respecta a la prueba de hipótesis, se calculó la U de Mann-Whitney, obteniendo una $U$ de 000 , lo cual indica que existen diferencias significativas entre ambas muestras y se apoya la hipótesis de investigación. En definitiva, las PyMEs comerciales y de servicios poseen un nivel de contribución empresarial medio-alto, lo que demuestra que las acciones implementadas por estas, asociadas a los ODS y la RSE aún durante la pandemia por la Covid, han coadyuvado al crecimiento empresarial y pone de manifiesto la importancia de estas acciones en las organizaciones. 


\section{REFERENCIAS}

Avendaño Ruiz, B., Sierra López, O., \& Lobo Rodríguez, M. (2017). Una estomación de la responsabilidad social empresarial en empresas hortofrutícolas de Baja California, México. Revista Mexicana de Ciencias Agrícolas, 6(1), 563-576. doi:10.29312/remexca.v6i3.639

Avendaño, C., \& William, R. (05 de octubre de 2013). Responsabilidad Social (RS) y Responsabilidad Social Corporativa (RSC): una nueva perspectiva para las empresas. Lasallista de Investigación, 10(1), 152-163. Obtenido de http://www.scielo.org.co/scielo.php?script=sci_arttext\&pid=S1794$44492013000100014 \& \operatorname{lng}=$ en\&nrm=iso\&tlng=es

Berlanga, V., \& Rubio, M. (2006). Clasificación de pruebas no paramétricas. Cómo aplicarlas en SPSS. REIRE, 5(2), 101-113. https://doi:10.1344/reire2012.5.2528

Cajiga, J. (2009). El concepto de Responsabilidad Social Empresarial. México: CEMEFI Centro Mexicano para la Filantropía.

Canal, R. (26 de junio de 2020). Deloitte.com. Obtenido de https://www2.deloitte.com/mx/es/pages/dnoticias/articles/COVID-19-una-prueba-deresponsabilidad-social.html

Celina, H., \& Campo, A., (2005). Aproximación al uso del coeficiente alfa de Cronbach. Revista $\begin{array}{llll}\text { Colombiana de } & \text { Psiquiatría, } & 34(4), & \text { 572-580. }\end{array}$ https://www.redalyc.org/articulo.oa?id=80634409

CEPAL. (2021). Cepal.org. Obtenido de https://www.cepal.org/es/temas/pymes/acercamicroempresas-pymes

De Miguel Corrales, M. (abril de 2011). Foroempresarias.com. Obtenido de http://www.foroempresarias.com/userfiles/archivos/Documentaci\%C3\%B3n/Gu\%C3\%ADa \%20RSE\%20PYME\%20DEFINITIVA.pdf

Enguix, T. (2021). Prácticas de responsabilidad social en las Pymes ¿Cuáles son sus beneficios? Revista PYMEAH, 37-40. Obtenido de http://plataforma.responsable.net/sites/default/files/practicas_de_rs_en_pymes.pdf

Esemanal. (18 de febrero de 2021). Esemanal.mx. Obtenido de https://esemanal.mx/2021/02/desafios-para-las-pymes-mexicanas-en-2021/

FEADPYME [Fundación para el Análisis Estratégico y Desarrollo de la Pequeña y Mediana Empresa]. (2018). Cepyme.es. Obtenido de https://www.cepyme.es/wpcontent/uploads/2018/11/informe-FAEDPYME-Espana-2018.pdf

Flores Campero, A. d. (2013). La Responsabilidad Social Empresarial en las pymes de Colombia Análisis a resultados de aplicación de herramienta de caracterización y Manual Cartilla par pequeños y medianos empresarios [Tesis de Especialización no publicada] Universidad Ean. Colombia.

Obtenido de https://repository.ean.edu.co/bitstream/handle/10882/5885/FlorezAndrea2013.pdf;jsessioni $\mathrm{d}=11 \mathrm{D} 27 \mathrm{D} 33 \mathrm{AAEB} 370 \mathrm{BEC} 4 \mathrm{EE} 2 \mathrm{C} 79 \mathrm{~A} 66 \mathrm{~A} 164$ ? sequence $=2$ 
GPTWR. (2020). Greatplacetowork.com.mx. Obtenido de https://greatplacetowork.com.mx/panorama-de-las-pymes-en-mexico-2020/

GRI [Global Reporting Initiative]/United Nations Global Compact/wbcsd [World Business Council for Sustainable Development]. (2016). Sdgcompass.org. Obtenido de https://sdgcompass.org/wp-content/uploads/2016/06/SDG_Compass_Spanish-one-pagerview.pdf

Hernández, R., Fernández, C., \& Baptista, M. (2014). Metodología de la Investigación. México: McGrawHill.

Herrera Madueño, J., Larrán Jorge, M., Lechuga Sancho, M. P., \& Martínez Martínez, D. (2016). Responsabilidad social en las pymes:. análisis exploratorio de factores explicativos. Revista de contabilidad, 19(1), 31-44. doi:http://dx.doi.org/10.1016/j.rcsar.2014.10.003

INEGI. (2020). Inegi.org.mx. Obtenido de https://www.inegi.org.mx/programas/ecovidie/2020/

INEGI. (2021). Inegi.org.mx. Obtenido de https://www.inegi.org.mx/contenidos/programas/edn/2020/doc/EDN2020Pres.pdf

Labelle, F., \& Navarrete Baez, F. (2018). Las prácticas de responsabilidad social empresarial en las pequeñas y medianas empresas en Jalisco y Quebec: ¿isomorfismos después del TLCAN? Investigación Administrativa, 47(121). Obtenido de http://www.scielo.org.mx/pdf/ia/v47n121/2448-7678-ia-121-00002.pdf

MISION SOSTENIBLE. (2019). Misionsostenible.com. Obtenido de https://misionsostenible.com/conoce-las-5-p-del-desarrollo-sostenible/

ONU. (2015). Obtenido de https://www.un.org/sustainabledevelopment/es/2015/09/la-asambleageneral-adopta-la-agenda-2030-para-el-desarrollo-sostenible/

Ortíz, A. (2015). Enfoques y métodos de investigación en las ciencias sociales y humanas. Bogotá, Colombia. Ediciones de la U.

Otzen, T. \& Manterola, C. (2017). Técnicas de Muestreo sobre una Población a Estudio. Int. J. Morphol, 35( 1 ), 227-232. http://dx.doi.org/10.4067/S0717-95022017000100037

Remacha, M. (2017). Empresa y objetivos de desarrollo sostenible. Cátedra CaixaBank de Responsabilidad Social Corporativa. Obtenido de https://media.iese.edu/upload/ST0438.pdf

Ricart, J. E., \& Rodríguez, M. Á. (2005). Media.iese.edu. Obtenido de https://media.iese.edu/research/pdfs/ESTUDIO-94.pdf

Roser Hernández, I., Cabrerizo Sanz, L., De la Cuesta González, M., \& Vázquez, O. (2005). Guía de la responsabilidad social corporativa para las pymes. Observatorio de Responsabilidad Social Corporativa/Fundación El Monte. Obtenido de https://observatoriorsc.org/wpcontent/uploads/2013/07/Guia_RSC_PYME.pdf

Senado de la República (2020). PyMEs, importante motor para el desarrollo económico nacional: MC. $\quad$ http://comunicacion.senado.gob.mx/index.php/informacion/boletines/47767PyMEs-importante-motor-para-el-desarrollo-economico-nacional-mc.html 
Urriolagoitia, L., Murillo, D., \& Lozano, J. (2009). RSE y pyme: del discurso a la implementación. Una perspectiva europea. Madrid: Dirección General de Política de la Pequeña y Mediana Empresa. Obtenido de http://www.ipyme.org/Publicaciones/RSE-PYME.pdf

Viteri Moya, J. (2010). Responsabilidad Social. Enfoque UTE, 1(1), 90-100. 